\title{
Discourse under control in ambiguous sentences
}

\author{
Vincenzo Moscati \\ Università degli studi di Siena
}

\section{Introduction}

A recent topic in language acquisition is children's comprehension of sentences containing multiple scope-bearing elements. The main problem posed by those sentences is that the relative scope of each operator might be reversed and children need to relate a single sentence to two distinct logic representations. Sometimes languages have means to reduce ambiguity, as in negative sentences, where quantifiers present a specification relative to their polarity. However, also this information needs to be acquired, and even if there is evidence that children are sensitive to polarity from early on (Thornton, 1994), several studies have shown that under certain circumstances, children interpret multiple-operator sentences differently from adults.

Initial findings by Musolino (1998) and Musolino et al. (2000) suggested that in an early developmental stage, scope assignment adheres to surface structure relations. This means that the surface c-command relations observable at PF are also respected at LF, resulting in an Isomorphic LF-PF mapping.

This view has been successively questioned by more recent studies (Gualmini 2004a, Husley et al. 2004, Kramer 2000) which challenge the conclusion that surface relations determine scope assignment. According to Husley et al. (2004), structural relations are not the only way to look at the phenomenon and a closer observation to the link between a sentence and the discourse can explain children's deviant interpretations. If one possible interpretation is contextually inappropriate, it will be disfavored, regardless of surface c-command relations between logic elements.

Additional evidence against Isomorphism comes from the study of modality. Moscati \& Gualmini (2008) looked at modal verbs, instead of nominal quantifiers, in negative sentences. Consider the examples in (1) and (2):

(1) The red ball cannot be with the yellow ball

a. it is not possible that the red ball is together with the yellow ball

b. *it is possible that the red ball is not together with the yellow ball

(2) L' Indiano non deve cavalcare l' ippopotamo

the Indian not must ride the hippopotamus

a. it is necessary that the Indian does not ride the hippopotamus

b. it is not necessary that the Indian rides the hippopotamus

The inverse scope readings for sentences (1) and (2) are given in (1a) and (2a). Such readings are fully target consistent and they are perfectly licit in the adult language. In the case of (1), the inverse-scope reading (1a) is the only one allowed in adult English while sentence (2) -with plain intonation- is instead ambiguous in Italian. According to the prediction of Isomorphism, readings 
(1a) and (2a) should be problematic. Contrary to this expectation, Moscati and Gualmini (2008) found that children do not have problems with those interpretations.

From those results, it seems that children already have an adult-like competence with respect to the interpretation of modals. However, there are cases in which children fail to give a correct interpretation. In sentences similar to (3), from Moscati and Gualmini (in press), children incorrectly select the meaning (3b), which is not allowed in adult English:

(3) you need not feed the zebra

a. it is not necessary to feed the zebra

b. *it is necessary not to feed the zebra

Although this sentence is unambiguous and its only interpretation is (3a), children show a preference for reading (3b). This case, where the forbidden reading is selected, is reminiscent of the deviant interpretations with nominal quantifiers initially reported in Musolino (1998):

(4) the detective didn't find some guys

a. There are some guys that the detective didn't find

b. *It is not the case that some guys were found

In certain cases, children interpret sentence (4) as in the paraphrase (4b), which is forbidden by the polarity of some. Following Husley et al. (2004), children choose this particular interpretation only under particular conditions. In fact, when the discourse made felicitous the reading (4a), deviant interpretation vanishes.

A natural way to look at sentence (3) is to verify if the discourse also plays a role in this case. The problem is how to capture discourse adherence. The Question-Answer-Requirement (QAR) proposed by Husley et.al (2004) seems to be a sufficiently explicit model to make empirical predictions and it will be introduced in the next section.

In section §.3. new data from Italian are presented, showing the existence of children's misinterpretations similar to those seen for sentence (3). This case will be used in a successive experiment to test the predictions of the QAR.

\section{Satisfaction of the Question-Answer-Requirement}

The general view that extra-linguistic context and subject expectations may favor particular interpretations is relatively uncontroversial. Early studies by Wason (1965) show that a negative sentence requires an additional processing with respect to a positive declarative. However, Wason also showed that the processing load diminishes if an appropriate visual context is provided, a conclusion supporting the idea that the logic processor is sensitive to the background.

What we can learn from this is that, every time we want to test children's comprehension of negative sentences, we should be aware of the extra work required by their cognitive system. Given that cognitive resources may be initially limited, children have to be in the best processing condition when called to process negative sentences.

This experimental requirement has been expressed by Crain ad Thornton (1998) through the need to satisfy the condition of plausible dissent. If we want to test the comprehension of a negative proposition $\neg(p)$, it will be easier to process if children have already considered (p).

In the view of Wason and Crain \& Thornton, the background plays a priming role, which can be considered a low-level automatic mechanism. However, it is not sufficient to restrict the context's role only to a contrastive function in the case of negative sentences. Sentence processing can also be influenced by the communicative function of language, and the discourse is another important factor to consider. 
Since Grice (1975), it is a fairly uncontroversial assumption that an utterance must be maximally informative. This consideration is particularly relevant in the case of ambiguous sentences. In fact, if more than one interpretation is allowed, we might expect that the more informative -the more contextually relevant- interpretation should be preferred. If this reasoning is correct, knowledge of the speaker's communicative intentions/needs can also drive the interpretative process.

This intuition can be captured if we find a way to cast a sentence within the communicative context, in a way to make it possible to evaluate its contribution to the discourse.

A recent proposal in this direction has been put forward by Husley al. (2004). This proposal is based on the assumption, common to many theories of communication, that every assertion has to be informative with regard to a salient question. If a sentence is perceived as out-of-topic or redundant, this sentence does not constitute a direct answer to the Question under Discussion (QUD). The felicity of a sentence can then be evaluated through its capacity to satisfy the QAR. This requirement presents a certain flexibility and the hearer can rescue the appropriateness of a given sentence trying to consider a different QUD in order to comply with the QAR. Only when no other QUD is plausible, then we have a pragmatic violation that makes the sentence infelicitous.

The QAR differs from the condition of plausible dissent in that it takes into account not only the hearer's own expectations but also the communicative frame. It has to be considered as a complementary constraint to take into account when comprehension is examined.

In the next section, we will consider experimental evidence suggesting that young children are very sensitive to the QAR and that an undervaluation of such a requirement in the experimental setting might bias the outcome of truth-value judgments.

\subsection{The early QAR bias}

Musolino (1998) claimed that children, in an early developmental stage, are unable to access the adult meaning of sentences such as (4). However, more recent studies have shown that the conclusion, that children lack the adult reading, is too strong. In fact, children fail to correctly interpret (4) only in special cases. When the adult interpretation satisfies the QAR, children performance is adult-like.

What remains to be explained is why and under which circumstances children access deviant nonadult readings. In one experiment reported in Gualmini (2004a), children were divided into two experimental groups, and each group heard a sentence with negation and the indefinite some. The story context was the same for the two groups, while the test sentences only differed in the choice of the lexical verb:

(5) the firefighter didn’t find some dwarves

(6) the firefighter didn't miss some dwarves

The two sentences were truth conditionally equivalent given the story they heard. This was about a firelighter playing hide-and-seek with four dwarves and, at the end of the story, the outcome of the search was that the firefighter found only two out of four dwarves. Given the story context and the outcome of the game, both (5) and (6) were true, since two dwarves were found and two were missed. However, Gualmini found that while children accepted sentence (5) around $90 \%$ of the time, they only accepted (6) at chance level.

The difference in the acceptance rate for (5) and (6) can be accounted for if we consider how well the two sentences answer the QUD primed by the story. Children know that the goal of hide-andseek is to find everybody. Therefore, the question has to be the following:

(7) Did the fire-fighter find all the dwarves? 
Now consider how the different interpretations of (5) and (6) answer the QUD in (7). Let us look first at (5):

QUD: Did the firefighter find all the dwarves?

(5) the firefighter didn't find some dwarves

a. There are some dwarves that were not found $\quad \rightarrow$ NO $\quad$ (relevant answer)

b. ${ }^{*}$ It is not the case that some dwarves were found $\rightarrow \mathrm{NO} \quad$ (relevant answer)

Here both interpretations are good (negative) answers to the QUD and the discourse does not pose any special pressure on the deviant non-isomorphic interpretation (5b). Children then accept the adult reading (5a) which is true. Consider now the case of (6):

QUD: Did the firefighter find all the dwarves?

(6) the firefighter didn't miss some dwarves

a. There are some dwarves that were not missed $\quad \rightarrow$ ? (uninformative answer)

b. ${ }^{*}$ It is not the case that some dwarves were missed $\rightarrow$ YES (relevant answer)

The adult reading (6a) only says that some of the dwarves were not missed: here we do not know if all of them were actually found. This reading is not relevant and it violates the QAR. In contrast, the non-adult reading (6b) is more informative, since it says that no dwarf was missed and it constitutes a good (affirmative) answer to the QUD in (7). Here the context forces the reading (6b), even if this is not allowed in adult English. According to Husley et. al (2004) children are more sensitive than adults to the contextual pressure and this will determine the choice of wrong interpretations.

The QAR makes predictions that are not limited to the case of nominal quantifiers. In the rest of this paper, we will try to determine if children are also sensitive to the QAR in negative modal sentences.

\section{Non-adult interpretations with modals}

In principle, any sentence with more than one logic element might be ambiguous between two interpretations and modal verbs, when combined with sentential negation, are no exception. If we look at languages whose modal system presents underdetermined forms with respect to the scope of negation, we can find cases of real ambiguity ${ }^{1}$, as in the following example from Italian:

(8) L'Indiano non deve cavalcare l'ippopotamo

the Indian not must ride the hippopotamus

a. it is necessary that the Indian doesn't ride the hippopotamus

b. it is not necessary that the Indian rides the hippopotamus

In (8) the modal dovere 'must' takes either wide or narrow scope with respect to negation and both interpretations (8a-b) are possible. By looking at sentences such as (8) we might discover whether children only have a narrower range of interpretations and if they lack covert operations, as predicted by Isomorphism. In Moscati and Gualmini (2008) we provided evidence that children do not have problems interpreting sentence (8) in accordance with the inverse scope reading (8a). However, (8) cannot reveal if children have interpretations unavailable for adults, given that adults have both the possible logic readings.

\footnotetext{
${ }^{1}$ Intonation can help the disambiguation. However, with a plain intonation, both interpretations are acceptable.
} 
A more telling case is given by English negative sentences with the modal need. Here language specific constraints related with the polarity of need exclude wide scope of the modal. Consider sentence (9):

(9) you need not feed the zebra

a. it is not necessary to feed the zebra

b. *it is necessary not to feed the zebra

Adults can interpret (9) only in accordance with reading (9a). However, when children are called to judge the truth conditions of (9), they interpret the sentence according to the meaning given in (9b) (Moscati \& Gualmini, in press). This result is somehow surprising and calls for an explanation. How can we explain this finding? Two possibilities come to mind: the first is that the logic component cannot access reading (9a), the second is that both readings are available, but the QUD is not satisfied by (9a). In the next section, we will look at a case similar to the one given in (9) and we will test children's comprehension with a standard Truth Value Judgment Task (TVJT).

\subsection{Deviant interpretations in restructuring domains}

The combination of a modal verb with negation might be unambiguous also in Italian. One example is given by restructuring contexts (Rizzi, 1982), when negation appears between the modal and the non-finite lexical verb. Here, only an isomorphic reading is allowed. Consider sentence (10):

(10) Il contadino può non dare le carote all' elefante the farmer can not give the carrots to-the elephant a. *it is not possible that the farmer gives carrots to the elephant

b. it is possible that the farmer doesn't give carrots to the elephant

This sentence only allows one reading, the permission not to give carrots to the elephant in (10b), while the stronger prohibitive reading given in (10a) is excluded. At this purpose, remember from the previous section that children, at least in English, find it difficult to associate a weaker permissive reading to sentences as (9).

A plausible hypothesis is that children also have problems in the understanding of $(10 \mathrm{~b})$, given that this meaning is logically equivalent to the one in (9a):

(11) Not $>$ necessary $\equiv$ possible $>$ not

If Italian children also misinterpret sentence (10), we have an indication that deviant interpretations are related to a particular meaning, the one in (11).

To verify this prediction, we tested 20 monolingual Italian speaking children (Age: 3;9 5;7. Mean 4;5) from two kindergartens in the Siena area using a TVJT (Crain \& Thornton, 1998). Each child heard 4 sentences similar to the one in (10), after a short story had been acted out using props and toys. At the end of the story, children were asked to judge if the sentence correctly described the situation just presented. In the case of sentence (10), for example, children heard the following story:

A farmer has to feed one tiger and two elephants with some turnips and carrots. However, the tiger says that it doesn't like carrots and for this reason it receives a turnip. Then the farmer goes to the elephants. They say that they don't have any preference and the farmer decides to give a turnip to the first elephant and a carrot to the second one. 
At this point, a puppet describes what happened in the story, uttering sentence (10). Since there is one elephant in the story without carrots, it follows that it is possible not to give carrots to the elephant: the reading (10b) is true. Instead, the interpretation (10a) is false since an elephant receives a carrot and no prohibition exists.

The result is that children tend to reject the target sentence, in accordance with the deviant interpretation (10a). They accept the sentence only $34 \%$ of time, in contrast with the adults in the control group who always accepted it. Results are reported in Table 1.

Table 1: Acceptance of target sentences. Adults: $n=10$. Children: $n=20$.

\begin{tabular}{|l|c|c|}
\hline Group & Adults & Children \\
\hline Acceptance & $40 / 40(100 \%)$ & $25 / 73(34 \%)$ \\
\hline
\end{tabular}

To exclude other factors which might have determined rejection of sentence (10), a series of controls were included within the experimental design. Between the target trials, four fillers/controls were inserted.

The first type of control (Type A) has been included to test whether children have problems with the modal potere (to be able to), and with the use of the definite determiner in presence of two elephants ${ }^{2}$. To illustrate, here I report one of the stories heard before the control sentence:

A king has some magic objects: two swords and a harp. The swords give the ability to run fast while the harp gives the ability to fly. However, the swords are very heavy and it is not easy to hold them. Also, the harp is a difficult instrument and not everyone can play it. Semola goes to the king and he first tries to play the harp. He has success and he is now able to fly. Then he tries to lift the first sword, but it is too heavy. Then he tries with the second one, and this time he manages to lift it and he runs away to an incredible speed.

At this point, the puppet describes the events with the following sentence:

(12) Semola può sollevare la spada

'Semola can lift the sword'

If children accept this sentence, they show that they have no particular problem with the modal and with the definite determiner.

In the second control (Type B), we gave children sentences with a negative operator and potere, but in this case, the negation linearly precedes the modal, reversing the order given in the target sentence (10). The following is one of the stories used in Type B controls:

A pilot wants to go out and he is torn between taking a motorbike or an F1 car. He decides to take the motorbike, but when he tries to climb on it, he falls down since it is too high. He tries again, but he falls another time. He changes his mind and finally he takes the car.

At the end of the story, the puppet utters the following statement, true under the previous context:

(13) Il pilota non può guidare la moto

the pilot not can drive the motorbike

a. it is not possible that the pilot drives the motorbike

b. *it is possible that the pilot drives the motorbike

\footnotetext{
${ }^{2}$ Definite determiners were also chosen in the target condition in order to avoid the insertion of another scope-bearing element as an indefinite.
} 
Again, children are expected to accept the sentence if they can compute the meaning of negation and the modal.

The results are that 11 children correctly accepted all the controls and two children committed only one mistake in Type B. Taken together, those 13 children gave the correct answer to at least 3/4 of the controls and their rate of correct answers is above chance level. If we include only the data from those 13 children, and we look at their acceptance of the target sentences, the results are not significantly different from the one presented in Table 1 . Also, children that have no problems with i) definite determiners, ii) the use of modal potere and with iii) negative modal sentences expressing prohibition still accept the target sentence only in $31.2 \%$ of the cases.

Table 2. Results for 13 children accepting at least $75 \%$ of controls.

\begin{tabular}{|l|l|l|l|}
\hline Condition & Type A & Type B & Target \\
\hline Acceptance & $26 / 26(100 \%)$ & $24 / 26(93 \%)$ & $15 / 48(31.2 \%)$ \\
\hline
\end{tabular}

If we compare the results of Table 1 and Table 2, the acceptance rate is about the same. This suggests that children's poor performance with sentence (10) it is not due to the choice of the determiner or to the presence of two logic operators. Children seems to judge sentence (10) False because they interpret it under the reading (10a), expressing a prohibition which is not verified in the story context. This conclusion is supported by the fact that every time the child rejected the target sentence we asked for an explanation. In the vast majority of the cases ${ }^{3}$, the explanation was consistent only with the reading (10a).

\section{Truth Value Judgments and the context: Experiment II}

The results of Experiment I are similar to the findings of Moscati and Gualmini (in press). Children find it problematic, also in Italian, to process modals in negative sentences when the meaning is not a prohibition but instead a weaker permissive reading. This result cannot be captured adopting Isomorphism (or its weaker version as presented in Musolino \& Lidz, (2006)) since children in the previous experiment prefer the non-isomorphic interpretation even when this is not allowed by the adult grammar.

Sentence (9) and (10) share few syntactic properties and they differ in the force of the modal as well in the scope it takes at LF. A syntactic explanation in general does not seem to be adequate to account for children's deviant interpretation for sentences like (9) and (10). Instead, the fact that the interaction between negation and the modal results in an equivalent logic representation (see 11) suggests that the problem relies on the semantic, or in the pragmatic.

In fact, the meaning might not be problematic per se but might be problematic given the story context. We already saw that if the story favors a certain QUD, children tend to choose an interpretation salient with respect to this QUD, even if this is not permitted in the adult grammar.

We have seen that for nominal quantifiers, children accept the adult interpretation of some only when this interpretation does not violate the QAR. Instead, whenever the sentence is not entirely appropriate in the discourse, children may accommodate the felicity conditions choosing the alternative interpretation.

This consideration is relevant also in the case at issue here. In fact, we cannot exclude that children fail to access the permissive reading in (9) and (10) because it is not consistent with the QUD made salient by the story. The goal of this second experiment is to assess if the QAR biases children interpretation also in the case of modals.

\footnotetext{
${ }^{3}$ There were only four inconsistent explanations, and those responses were excluded from the count (for example: because elephants are strange.)
} 
A problem for the QAR account is that it is not easy to assess what is the actual question selected by the subject. A way to overcome this problem is to state explicitly the QUD, a solution which has been adopted in the experimental design.

In this experiment, we used the same 4 target sentences and the same 4 stories as the previous one, but this time the target sentence is presented after a question is asked to the puppet. In order to evaluate the role of the QAR, we use two different kinds of questions: in the fist condition, the QUD made felicitous both interpretations for the target sentence, while in the second condition, only the deviant reading permits the QAR to be satisfied.

As in the case of nominal quantifiers, we expect that if both readings are appropriate (Condition 1), the adult reading will be selected, since this is the interpretation that minimizes violations. Conversely, when only the deviant reading satisfies the QAR (Condition 2), we expect a competition between this discursive requirement and the syntactic constraints. If the child decides to avoid violations of the QAR, he will select the pragmatically appropriate interpretation even if this violates the syntax.

The only difference between the first and the second experiment is that before the target sentence, the puppet heard a question. Below an exchange is reported to illustrate the first experimental condition:

QUD1- First Experimenter:

(14) il contadino deve dare la carote all' elefante?

'Must the farmer give carrots to the elephant?'

ANSWER- Second Experimenter, manipulating the puppet:

(15) Il contadino può non dare le carote all'elefante

a. *it is not possible that the farmer gives carrots to the elephant $\rightarrow$ NO (relevant answer)

b. it is possible that the farmer doesn't give carrots to the elephant $\rightarrow$ NO (relevant answer)

In this condition both interpretations represent a good (negative) answer to the QUD. Either the prohibitive reading (15a) or the permissive reading (15b) entail that it is not necessary to give the carrot to the elephant: the QAR is satisfied by any interpretation of (15). Here, we expect that if children have the adult interpretation (15b) they will choose this reading. Since (15b) is true in the story context, children should accept the target sentence.

In the second condition, we varied the QUD in a way that only the deviant interpretation (15a) satisfies the QAR.

QUD2- First Experimenter:

(16) il contadino può dare la carote all' elefante?

'Can the farmer give carrots to the elephant?'

ANSWER- Second Experimenter, manipulating the puppet:

(17) Il contadino può non dare le carote all'elefante

a. *it is not possible that the farmer gives carrots to the elephant $\rightarrow$ NO (relevant answer)

b. it is possible that the farmer doesn't give carrots to the elephant $\rightarrow$ ? (uninformative answ.)

In (17) the modal potere 'can' was used instead of dovere 'must' in the QUD and here the only informative answer is (17a), expressing the prohibition to give carrots to the elephant. The other reading, the adult $(17 \mathrm{~b})$, is uninformative since it only states the possibility not to give carrots to the elephant. The context created by the QUD2 adds discursive pressure, which will favor the non-adult interpretation (17a). Since this interpretation is false given the story, children are expected to reject the target sentence. We then have a clear prediction: children will accept the sentence in the first condition but will reject it in the second condition. 
The experimental procedure is that each child heard the same 4 stories as in the previous experiment, but this time he heard at the end of the story the QUD1 or the QUD2, randomized in a way that subjects heard two questions of each kind. The participants were 16 monolingual Italianspeaking children aged between $4 ; 0$ and 5;5 (mean 4;9) ${ }^{4}$.

Looking at the results (Table 3), the acceptance rate for the two QUD is nearly the same and it is even higher for QUD2. The predictions of the QAR are not confirmed and instead, we found a slightly higher acceptance in condition 2 .

Table 3. Acceptance for the target sentence in response to QUD1 and QUD2

\begin{tabular}{|l|c|c|}
\hline & QUD 1 & QUD 2 \\
\hline Acceptance & $8 / 26(30.7 \%)$ & $10 / 25 \quad(40 \%)$ \\
\hline
\end{tabular}

Children in general show no sensitivity to variations of the QUD and the interpretation does not seem to be affected by the QAR. In the absence of contrary evidence, we can assume that the adult logic representation for the target sentences is harder to acquire and we have no evidence that at this developmental stage this reading is already available to the child.

\section{General discussion}

The first experiment confirms the findings presented in Moscati and Gualmini (in press) showing that Italian children also have difficulties with permissive reading in negative sentences. The results indicate that the pattern uncovered in Moscati and Gualmini is not language specific but seems to reflect a general developmental stage.

In order to capture the data and to unify this phenomenon with a similar deviance found with nominal quantifiers, in experiment II we explored the possibility that the pragmatic conditions, related to the experimental setting, bias children's response.

Extending the proposal by Husley et al. (2004), the role of the QAR was tested in experiment II. However, the results indicate that variation of the QUD, if explicitly asked, do not significantly affect children interpretation of the target sentence.

It is possible that the QUD, once created by the story, cannot be easily manipulated in an explicit way and that this masks the role of the QAR. It is also possible that subjects stop paying attention to the changes in the modal used in the QUD. This problem can easily be overcome using different groups of subjects for each condition, instead of adopting a design within subjects as in experiment II. More investigation is required to assess the role of the pragmatic context in the interpretation of multiple-operators sentences; however, in absence of empirical support, it is safer to assume that the discourse is not a key factor.

How can we explain then, the deviant readings for sentences (9) and (10)? A way to look at the pattern is to place the possible interpretations along a scale created by the strength of each interpretation's truth conditions. Consider the possible readings for (10), repeated here as (18):

(18) Il contadino può non dare le carote all'elefante
a. ${ }^{*}$ not $>$ possible
b. possible $>$ not

interpretation (18a) is stronger than (18b) since (18a) entails (18b) while the inverse entailment does not hold:

(19) not $>$ possible $\rightarrow$ possible $>$ not

\footnotetext{
${ }^{4}$ Two children gave unintellegible resonses in both conditions. Those trials are excluded from the count.
} 
(20) possible not -- $\rightarrow$ not $>$ possible

A conclusion that can be draw from the results of Experiment I, also consistent with the findings of Moscati and Gualmini (in press), is that if a sentence permits two logical interpretations, a bias toward the stronger interpretation exists in early grammar.

This result is also consistent with general considerations related to learnability (Berwick, 1985) and with the Semantic Subset Principle proposed by Crain et. al (1994).

However, the present study only considered one way of looking at the contextual background. Even if no role associated with the discourse has been detected here in the case of modals, further investigation is needed to exclude the role of contextual pressure on the selection of deviant interpretations.

\section{References}

Berwick, Robert. 1985. The Acquisition of Syntactic Knowledge. Cambridge, MA: The MIT Press.

Crain, Stephen, Laura Conway and W. Ni. 1994. "Learning, Parsing, and Modularity" in Perspectives on Sentence Processing edited by Clifton, Charles, Lyn Frazier and Keith Rayner, 443-467, Lawrence Erlbaum Associate, Hillsdale, NJ.

Crain, Stephen and Rosalind Thornton. 1998. Investigations in universal grammar: a guide to experiments on the acquisition of syntax and semantics. Cambridge, Mass.: MIT Press.

Grice, Paul. 1975. Logic and conversation. In Syntax and Semantics, ed. Peter Cole and James Morgan, 41-58. New York: Academic Press.

Gualmini, Andrea. 2004a. Some knowledge children don’t lack. Linguistics 42: 957-982.

Hintikka, Jakko. 1962. Knowledge and Belief. Ithaca, NY: Cornell University Press.

Horn, Laurence R. 1989. A natural history of negation. Chicago: University of Chicago Press.

Hulsey, Sara, Valentine Hacquard, Danny Fox and Andrea Gualmini. 2004. The Question-Answer requirement and scope assignment. Plato's problem: problems in language acquisition, ed. by Aniko Csirmaz, Andrea Gualmini and Andrew Nevins, 71-90. Cambridge, Mass.

Krämer, Irene. 2000. Interpreting Indefinites. Ponsen \& Looijen, Wageningen.

Ladusaw, William. 1979. Polarity Sensitivity as Inherent Scope Relations. PhD Dissertation, University of Texas at Austin.

Lewis, David. 1993. Counterfactuals. Oxford: Basil Blackwell.

May, Robert. 1985. Logical form : its structure and derivation. Cambridge, Mass.: MIT Press.

Moscati, Vincenzo. 2008. Negative Chains and LF Negation Raising, in Bisetto, A. e Barbieri, F.E. (ed.), Proceedings of XXXIII Incontro di Grammatica Generativa, Bologna, Italy. 
Moscati, Vincenzo. 2006, The Scope of negation. Doctoral Dissertation, Università degli Studi di Siena.

Moscati, Vincenzo and Andrea Gualmini. 2008. More Facts that Isomorphism Cannot Explain, in T. Friedman and M. Gibson (eds.) Proceedings of SALT XVII, CLC Publications, Cornell University, Ithaca.

Moscati, Vincenzo and Andrea Gualmini. in press. The Early Steps of Modal and Negation Interactions: evidence from child Italian. Romance Languages and Linguistic Theory. Ed. Enoch Aboh, Elisabeth van der Linden, Josep Quer \& Petra Sleeman. Amsterdam, John Benjamins.

Musolino, Julien. 1998. Universal Grammar and the Acquisition of Semantic Knowledge: an Experimental Investigation into the Acquisition of Quantifier-Negation Interaction in English, Doctoral Dissertation, University of Maryland at College Park.

Musolino, Julien and Jeffrey Lidz. 2006. "Why Children Aren't Universally Successful with Quantification", Linguistics, 44, 817-852.

Musolino, Julien, Stephen Crain and Rosalind Thornton. 2000. Navigating negative quantificational space. Linguistics, 38.1-32.

Palmer, R. Frank. 1986. Mood and Modality. Cambridge: Cambridge University Press.

Rizzi, Luigi. 1982. Issues in Italian Syntax. Foris, Dordrecht

Szabolcsi, Anna. 2004. Positive polarity-negative polarity. Natural Language and Linguistic Theory. 22/2: 409-452.

Thornton, Rosalind. 1994. Children's Negative Questions: A Production/Comprehension Asymmetry. Proceedings of ESCOL 306-317.

Wason, Peter. 1965. The contexts of plausible denial. Journal of Verbal Learning and Verbal Behavior, 4:7-11. 